\title{
Influencing Factors of the Power Fluctuation on the Ultra High Voltage Transmission Line Caused by Faults at the Remote Ends of the Interconnected Grid
}

\author{
Zhi An, Ansi Wang, Huadong Sun, Yong Tang \\ China Electric Power Research Institute, Beijing, China \\ Email: thuaz07@gmail.com
}

Received January 2014

\begin{abstract}
After the North China grid and the Central China grid get into connection with the UHVAC demonstration, a new phenomenon is discovered according to some simulations. That is, the faults at the remote end of the UHV interconnected grid will result in significant power fluctuation and voltage drop on the UHV transmission line and even system splitting. But the faults near the UHV line only have marginal effects. Further, the simulation results also indicate that the short-circuit current of the buses near the UHV line is larger than that of the buses far away from the UHV line. This phenomenon is divergent from the traditional view. In this paper, the detail will be introduced, and the factors influencing the system stability after faults are presented and analyzed. The results indicate that transmission power of the UHV line and of the lines between the remote end and the major grid influence the fluctuation on UHV line. The load model and the grid structure of the remote end also have effect on it. Finally, corresponding control scheme is presented to improve the operation conditions of the UHV interconnected grid and ensure its security and stability.
\end{abstract}

\section{Keywords}

Ultra High Voltage; UHV Interconnected Grid; Faults at Remote End; Power Fluctuation

\section{Introduction}

Along with the operation of Changzhi-Nanyang-Jingmen UHVAC (Ultra high voltage alternating current) demonstration project, the North China grid and the Central China grid got linked by a UHV transmission line and became a typical long chain system with weak ties [1]-[5]. Traditionally, it was considered that there would be greater impact when there was larger short-circuit current and it was closer to the fault bus [6]. However, opposite results were showed in recent simulation. Under certain modes of operation, it would result in significant power fluctuation and voltage drop on the Changzhi-Nanyang UHV line when three-phase instantaneous short circuit faults occurred at Bus Jianshan and its near buses in Sichuan grid which is located at the remote end of UHV Interconnected Grid, while the same faults occurring at Bus Jiangling in Hubei grid would not cause ob- 
vious effect. Considering that Bus Jiangling has larger short-circuit current and it is closer to the UHV line, this phenomenon is divergent with traditional view and is a new problem after the two large regional grids interconnected. It limits the capacity of UHV transmission line and threatens the security and stability of the UHV interconnected grid as well.

This paper analyzed the factors affecting the power fluctuation and voltage drop on the UHV transmission line in four aspects and presented corresponding control scheme to improve the operation conditions of the UHV interconnected grid and ensure its security and stability.

\section{The Phenomenon of the Fluctuation}

Figure 1 shows the schematic of the UHV interconnected grid. The Central China grid includes five parts: Sichuan, Chongqing, Hunan, Jiangxi and Henan. Each part is connected with $500 \mathrm{kV}$ AC transmission lines, except that there is another Nanyang (in Henan)-Jingmen (in Hubei) UHV line connecting Henan and Hubei. The North China grid and the Central China grid are connected with the Changzhi (in Shanxi)-Nanyang UHV line.

Under some modes of operation, significant power fluctuation and voltage drop could be observed when short circuit faults occurred at $500 \mathrm{kV}$ Bus Jianshan in Sichuan grid which is located at the remote end of UHV interconnected grid. On the contrary, the same faults occurring at $500 \mathrm{kV}$ Bus Jiangling in Hubei grid caused much smaller effects though it has larger short-circuit current with shorter distance from UHV transmission line. Figures 2 and 3 show the power and voltage curve respectively while $0.1 \mathrm{~s}$ three-phase short circuit faults occurred at main buses in different regions. The typical mode of operation: 2200 MW from Central China to North China on Nanyang-Changzhi UHV line, 2440 MW from Sichuan to Hubei.

It can be observed that the power fluctuation and voltage drop are significant while the fault occurs at Bus Jianshan, while they are slight in other situations. To ensure security and stability of UHV interconnected grid, it's necessary to figure out the factors which affect this phenomenon.

\section{Analysis on Factors Which Affects the Fluctuation}

\subsection{Transmission Power on UHV Line}

In this section, the influence of transmission power on UHV line will be studied through simulation. The initial

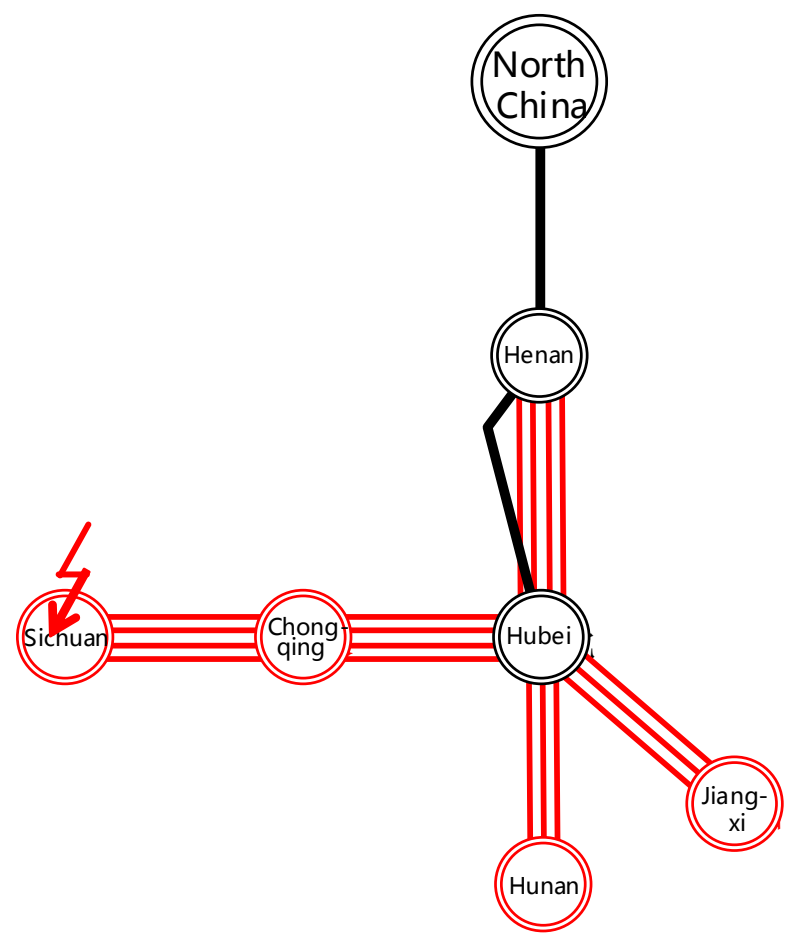

Figure 1. The schematic of UHV interconnected grid. 


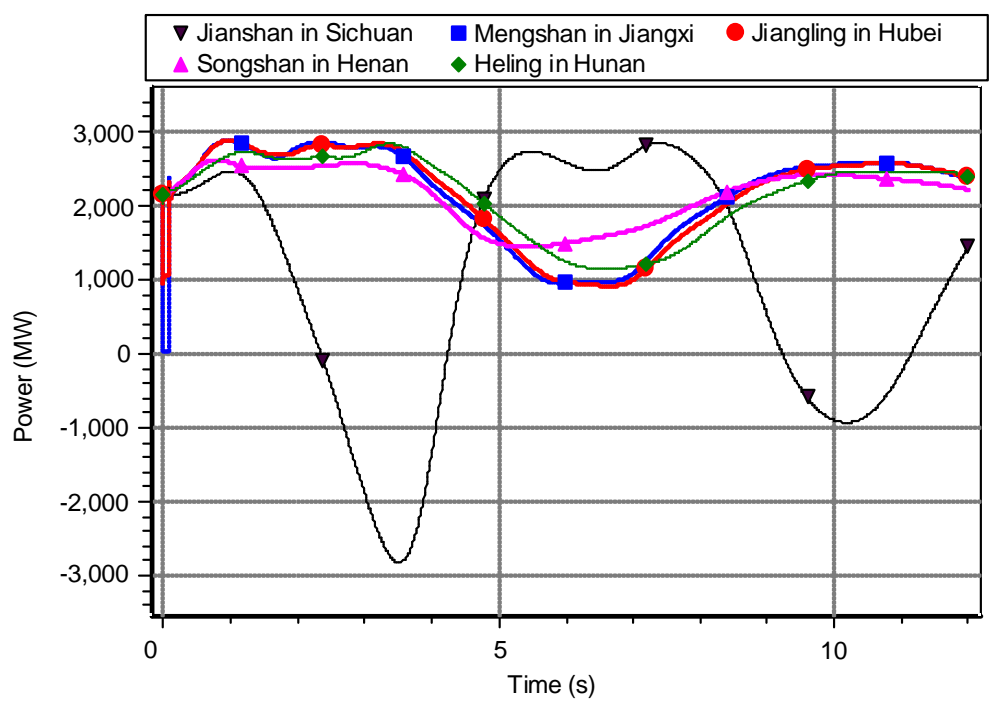

Figure 2. The transmission power of UHV Changzhi-Nanyang line.

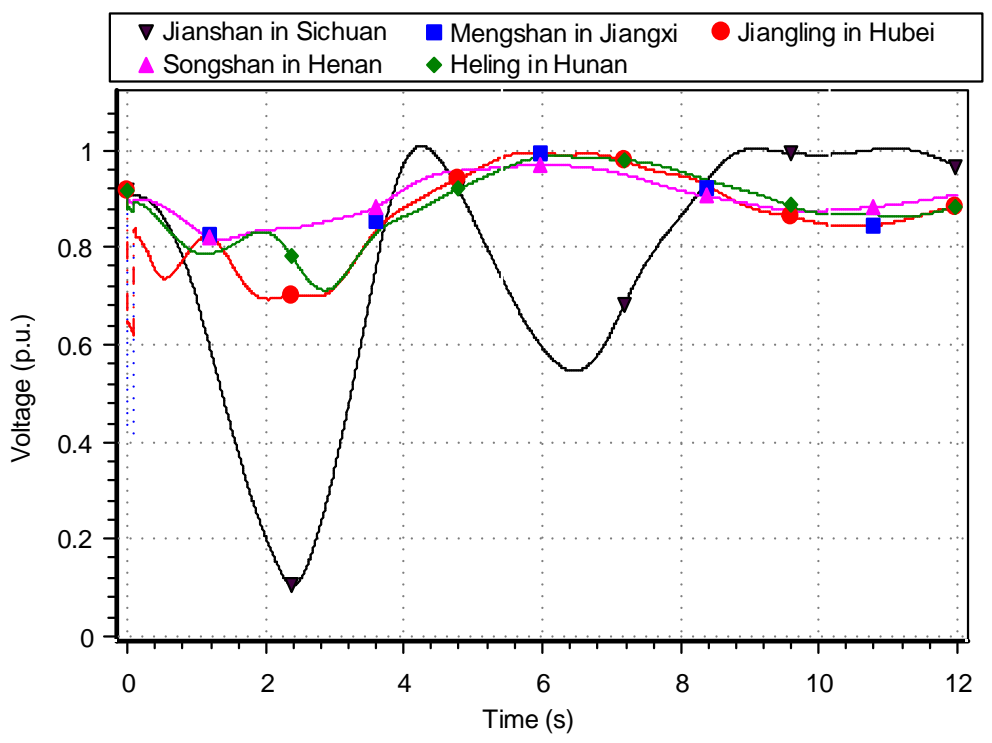

Figure 3. The voltage of $1000 \mathrm{kV}$ bus Changzhi.

transmission power will be modified by change unit commitment, i.e., to shutdown generators in Hubei and turn on generators in Shanxi. Setting different levels of initial transmission power and simulating $0.1 \mathrm{~s}$ three-phase short circuit fault at Bus Jianshan respectively, power curves are showed in Figure 4.

It can be concluded that the system will keep stable when initial transmission power is lower than $1650 \mathrm{MW}$. By reducing transmission power on UHV line, there will be larger stability margin, thus the UHV line will not oscillate significantly.

\subsection{Transmission Power from Sichuan Grid to Major Central China Grid}

The concerned issue in this paper manifests as impacting disturbance occurring at remote ends of system causes power fluctuation and even angle instability on UHV line. Thus changes of transmission power from Sichuan (the remote end) to major Central China grid would have effects on the fluctuation.

Changing power transmitted from Sichuan grid, the effects on system stability will be analyzed. Figure 5 shows the power curve of UHV line at different level of initial power transmitted from Sichuan. This mode of operation is 5800 MW from Nanyang to Changzhi and 4000 MW from Sichuan to Hubei. 


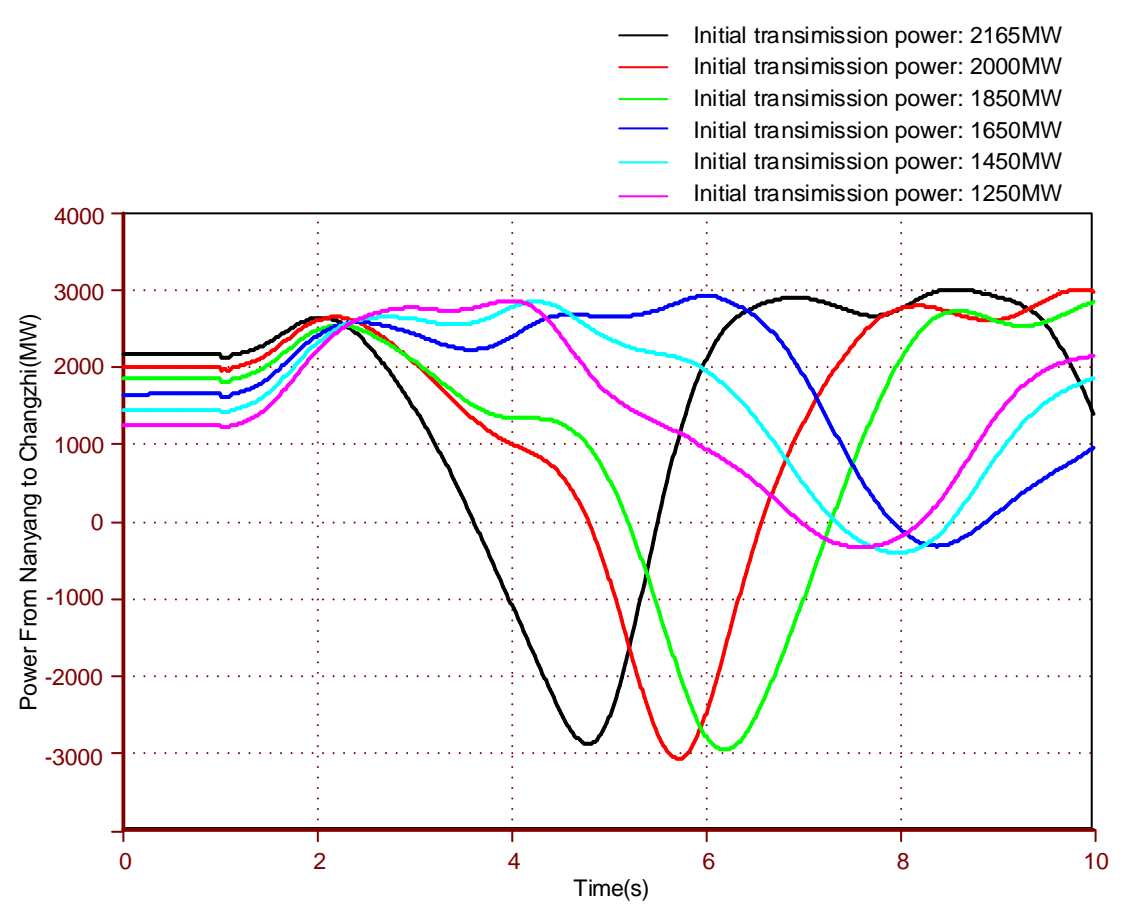

Figure 4. The power of UHV line at different initial power.

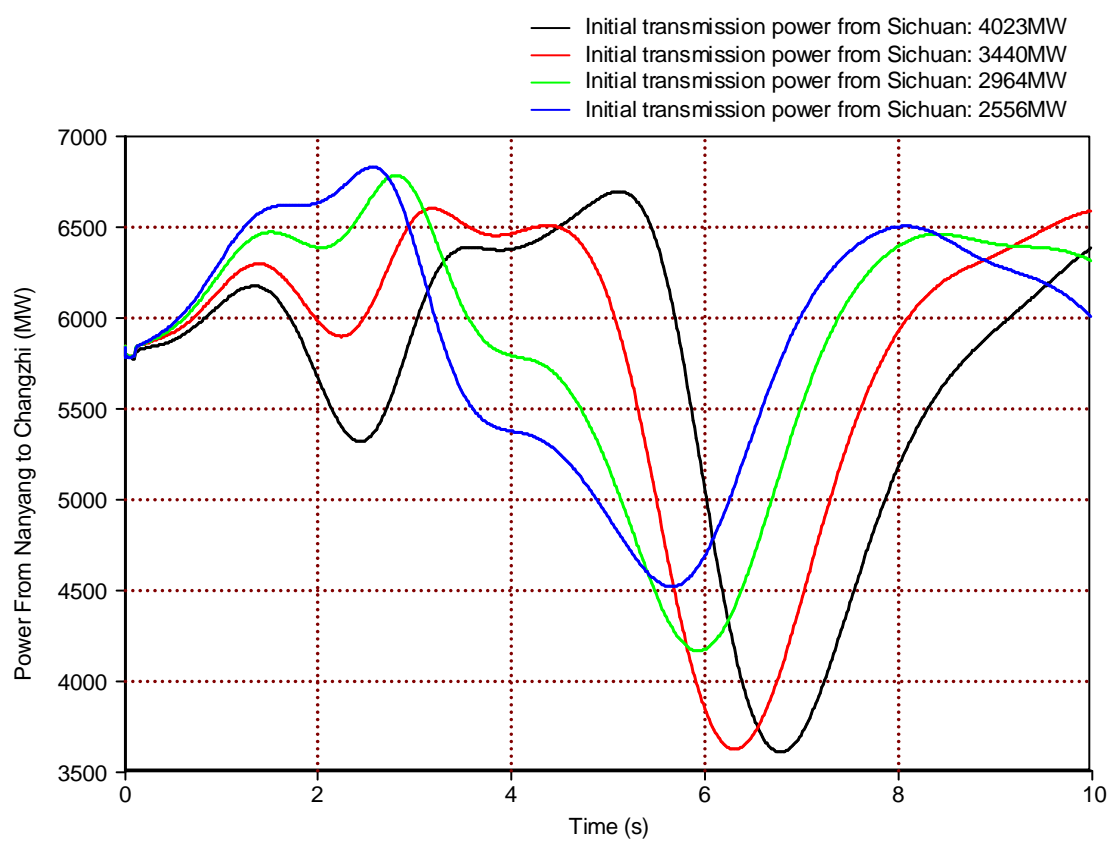

Figure 5. The power of UHV line at different initial power transmitted from Sichuan.

It can be concluded that the fluctuation on UHV line significantly becomes slight along with Sichuan-Hubei initial power reducing, thus the stability of interconnected grid improves. In situations of the same fault ( $0.1 \mathrm{~s}$ three-phase short circuit fault on $500 \mathrm{kV}$ Bus Jianshan), the impact on the system will significantly decrease by reducing the initial power transmitted from the remote end Sichuan.

After the fault at Bus Jianshan, unbalanced power transfers outward from Sichuan grid, the angle difference of Sichuan and Hubei increases. Along with initial power reducing, the initial angle difference decreases, and unbalanced power doesn't impact so strong as before, thus the stability level of system keeps relatively high. 


\subsection{The Load Model}

Load is one of the most important components of power system. Its characteristics have important influence on security and stability features of the system [7]-[9]. Generally power system load is divided into 2 categories: static load and dynamic load. The static load mainly refers to load without rotating equipment, and dynamic load refers to load with them [10] [11]. As there are differences in characteristics among various types of load models, simulation results will be affected when using different models.

Aiming at the impact caused by faults at Bus Jianshan, the load model of Sichuan grid will be selectively analyzed. The load model is $60 \% \mathrm{Z}$ (constant impedance) $+40 \% \mathrm{M}$ (electromotor) in synthesis load model at present [12]. Table 1 shows the stability result when using different types of load model.

When $100 \% \mathrm{Z}$ or $100 \%$ I (constant current) load model is used in Sichuan grid, the system keeps stable after faults. However, the stability features totally change after the load model becomes $100 \% \mathrm{P}$ (constant power). The fluctuation center transfers from UHV line to neighborhood of Chongqing, as Figure 6 shows.

In this situation, though there isn't the secondary impact of load releasing unbalanced power, the $100 \% \mathrm{P}$ load has terrible disadvantage for voltage recovery. The voltage of Sichuan grid which transmits power to other regions will remain low along with continuous power transfer. Therefore, the Sichuan grid is finally out of synchronism with major Central China grid.

$60 \% \mathrm{Z}+40 \% \mathrm{M}$ load model is used at present. When the ratio of electromotor increases to $60 \%$, the stability level will significantly decrease. Figure 7 compares the voltage curves of $1000 \mathrm{kV}$ Bus Nanyang using the two types of load model.

With the ratio of electromotor of the power sending end (Sichuan grid) increasing, the dynamic voltage support becomes worse and the voltage gets lower. The spreading of the low voltage weakens the stability of system. The transfer of impacting power intensifies the effect and makes the system even worse.

In conclusion, load model affects the system stability in two aspects. One is the stability limit, the other is the secondary impact of unbalanced power released by load. The influence on stability limit is mainly reflected in the voltage drop after faults caused by constant power load. The secondary impact mainly refers to that the unbalanced power released by model along with the voltage drop increasing transmission pressure of the sending end. The two aspects interact and are not easy to separate, however, one will play a leading role in certain situations.

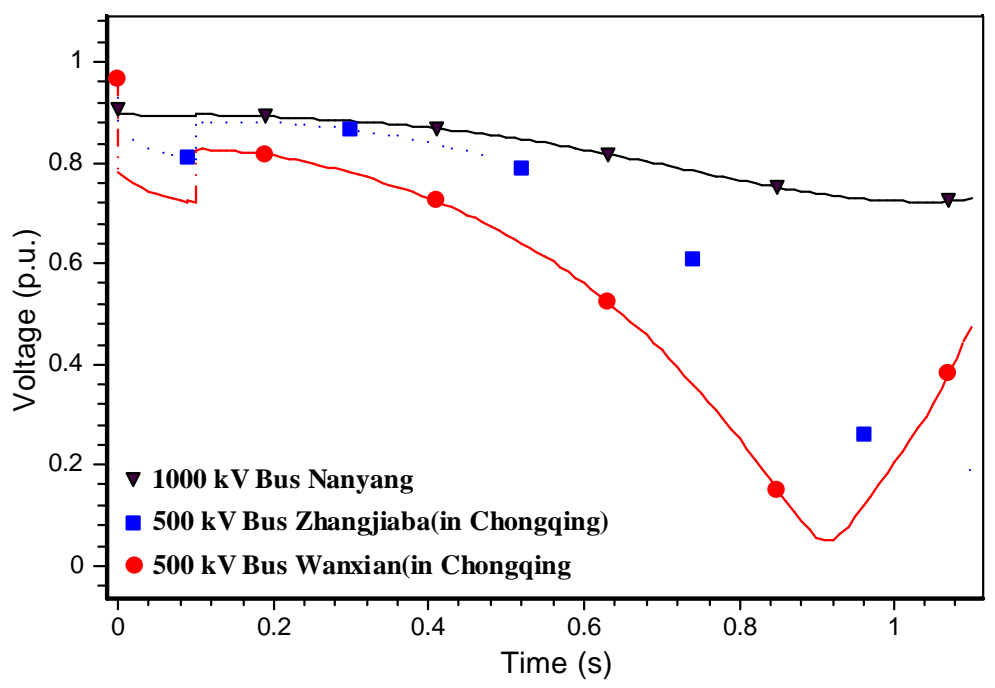

Figure 6. Voltage curves (100\% P load in Sichuan).

Table 1. Stability results using different load models in Sichuan.

\begin{tabular}{cccccc}
\hline Load model & $100 \% \mathrm{Z}$ & $100 \% \mathrm{I}$ & $100 \% P$ & $60 \% \mathrm{Z}+40 \% P$ & $40 \% \mathrm{Z}+60 \% \mathrm{M}$ \\
\hline Stability result & Stable & Stable & Unstable & Unstable & Unstable \\
\hline
\end{tabular}




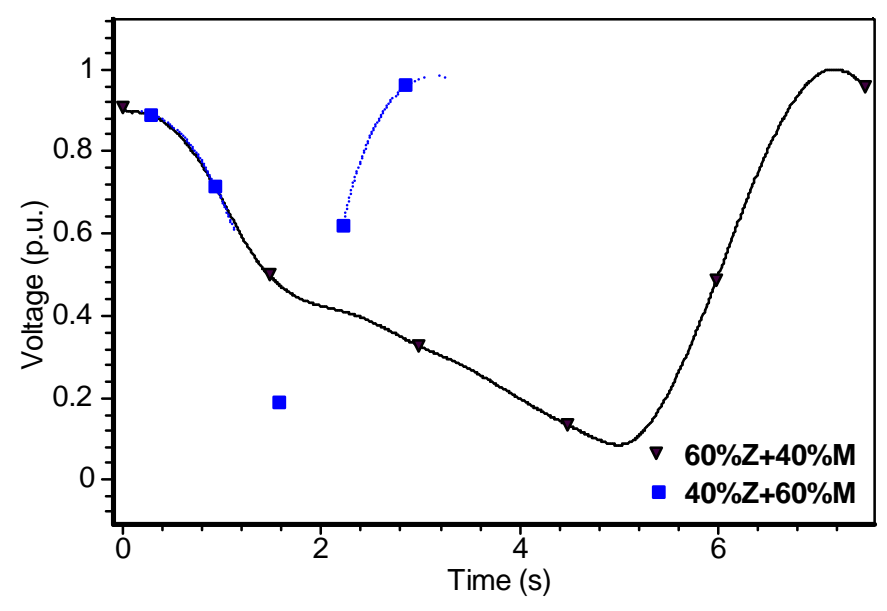

Figure 7. Voltage curves of bus Nanyang with different load models.

\subsection{The Grid Structure}

At present, Bus Jianshan is connected to Bus Taoxiang with four circuits, which results in a large short-circuit capacity of Bus Jianshan and large impact during the fault. By cutting off two-circuit line, the same fault in Jianshan would not have so serious effects to the system stability as before [13]. Figure 8 shows voltage curve of $1000 \mathrm{kV}$ Bus Changzhi before and after the structure changing.

Cutting off two-circuit line puts longer electrical distance between Jianshan and Taoxiang and decreases the short-circuit capacity, thus it weakens the impact to the system stability.

\section{The Control Measures}

According to the analysis above, there are two sorts of measures to keep the system stable: one is to reduce effects of faults to weaken its impact to the UHV line, and the other is to improve stability of the UHV line to enhance its ability to resist heavier impact.

\subsection{Reducing Effects of Faults}

To reduce effects of faults, three species of methods can be adopted.

1) Adjustment of the unit commitment

Widening of Angle difference is closely related to unbalanced power in system after faults. Advantages exist for reducing the effects to increase the rotational inertia of generators in the sending end of grid, thus it benefits the system stability.

Increasing output of generators with larger inertia and decreasing output of those with smaller inertia in the meantime was one feasible method to increase the rotational inertia of Sichuan grid, by which the flow of sections remained unchanged. Figure 9 shows the power curves of the UHV line before and after the adjustment of the unit commitment. It can be seen that the amplitude of power fluctuation significantly decreases, thus the system stability improves.

2) Reducing the short-circuit capacity

In Section 3.4, relative methods have been discussed. The short-circuit capacity can be reduced by cutting of two-circuit line from Jianshan to Taoxiang, through which the system stability improves.

Similar methods like cutting off one circuit of Jishan-Dongpo or shutting down generators nearby Jianshan are also effective to improve the situation.

3) Enhancing dynamic reactive power support

By adding SVC or other dynamic reactive power compensations, voltages of the area near the fault bus recover sooner. Therefore unbalanced power is released while system voltage drop decreases, thus the impact to the system reduces.

Figure 10 shows the power curves of the UHV line that the SVC capacity of Bus Taoxiang is 180 MVar, 270 MVar and 360 MVar. 


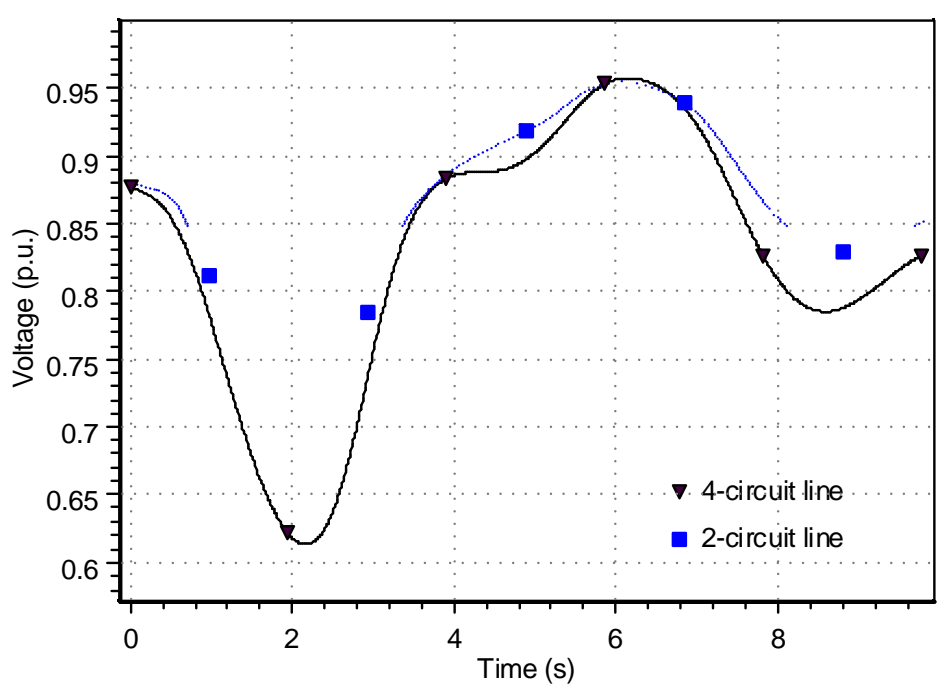

Figure 8. Voltage curves of bus Changzhi with different gridstructures.

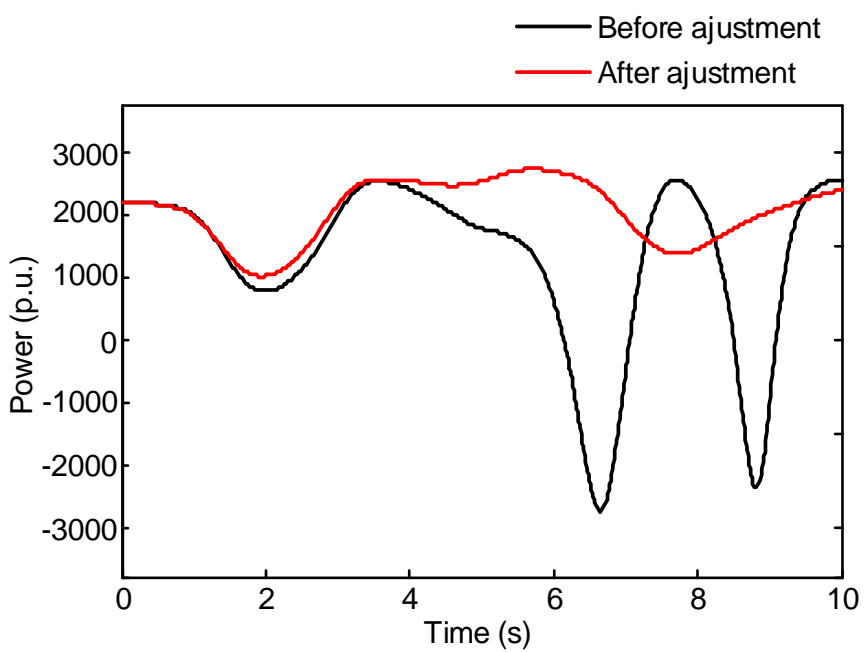

Figure 9. Powerof the UHV line with different unit commitment.

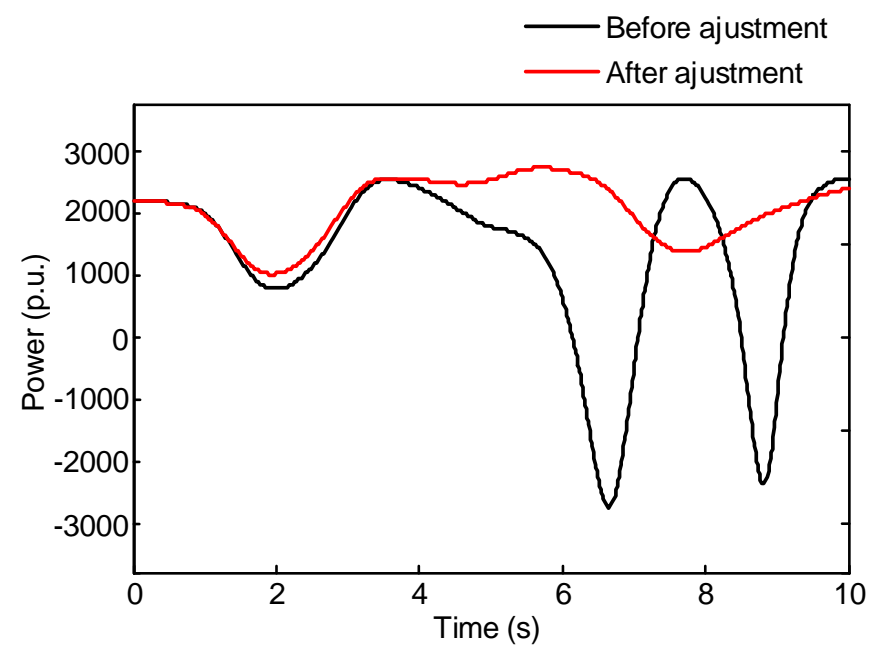

Figure 10. Powerof the UHV line with different SVC capacity. 
When the SVC capacity increases to 270 MVar, the amplitude of power fluctuation has become small. So the system stability may be improved by adding more SVCs.

\subsection{Enhancing the UHV Line}

Reducing the original transmission power or adding SVCs at UHV buses can enhance the ability of UHV line to resist impact by increasing stability margin and improve the ability of voltage recovery in transient process of the UHV line, though the feasibility of these methods is smaller than methods in Section 4.1.

\section{Conclusion}

This paper described the new phenomenon that faults at remote ends of the UHV interconnected grid would cause significant power fluctuation and voltage drop on the UHV transmission line while faults at buses near the UHV line with larger short-circuit current only have marginal effects. Then factors affecting the phenomenon were analyzed through simulation, which include the transmission power on the UHV line, the power transmitted from Sichuan to major Central China grid, the load model and the grid structure. Finally corresponding methods to improve the system stability were presented and the effectiveness of those methods was verified.

\section{Acknowledgements}

This paper is supported by Major Projects on Planning and Operation Control of Large Scale Grid (SGCCMPLG001-2012) of State Grid Corporation of China.

\section{References}

[1] Liu, Z.Y. (2005) Ultra-High Voltage Grid. China Economic Publishing House, Beijing.

[2] Shu, Y.B. and Zhang, W.L. (2007) Research of Key Technologies for UHV Transmission. Proceedings of the CSEE, 27, 1-6.

[3] Shu, Y.B., Zhang, W.L., Zhou, X.X., Tang, Y. and Guo, Q. (2007) Security Evaluation of UHV Synchronized Power Grid. Proceedings of the CSEE, 27, 1-6.

[4] Zhang, W.L., Zhou, X.X., Yin, Y.H., Tang, Y. and Guo, Q. (2010) Composition and Security Analysis of "North ChinaCentral China-East China” UHV Synchronous Power Grid. Proceedings of the CSEE, 30, 1-5.

[5] Guo, J.B., Zhou, J., Guo, Q., et al. (2011) Digital and Analog Hybrid Simulation of Interconnected UHVAC/UHVDC Transmission System from North China via Central China to East China. Power System Technology, 35, 55-59.

[6] Sun, Q.Z., Cai, Z.X., Li, A.M., Li, L. and Wu, K.C. (2009) A Short-Circuit Current Over-Limited Mechanism of 500 kV Power System and the Adaptability of Limiting Measures. Automation of Electric Power Systems, 33, 92-96.

[7] Chen, F., Li, X.R., Chen, H.H., Tang, W.W. and Liu, Y.Y. (2004) Effect of Induction Motor Model Structure and Parameters on Simulating Computation of Electric Power System Transient Stability. Electric Power Automation Equipment, 24, 29-33.

[8] Han, D., Ma, J., He, R.M., Yue, C.Y. and Zhang, J. (2008) Effect of Uncertainty in Load Model on Power System Dynamic Simulation. Proceedings of the CSEE, 28, 69-74.

[9] Yang, J.X. and Ma, J. (2010) Impact of Load Model on the Error of Power System Hybrid Simulation. Journal of North China Electric Power University, 37, 38-42.

[10] Zhang, H.B., Tang, Y., Zhang, D.X. and Hou, J.X. (2007) Present Situation and Prospect of Load Modeling Technique. Power System Technology, 31, 6-10.

[11] Liu, J.Q., Tao, J.Q., Xu, X.W. and Zhang, H.P. (2007) A Survey on Research of Load Model for Stability Analysis in Foreign Countries. Power System Technology, 31, 11-15.

[12] Zhang, H.B., Tang, Y., Zhang, D.X., et al. (2007) Analysis on Effects of Different Load Models on Transmitting Capacity of Northeast China Power Grid. Power System Technology, 31, 55-58.

[13] Zhang, Y.K., Cai, Z.X., Li, A.M. and Xu, M. (2009) An Optimization Algorithm for Short-Circuit Current Limitation of 500 kV Power Grid by Adjusting Power Grid Configuration. Automation of Electric Power Systems, 33, 34-39. 